\title{
Shear rate specific blood viscosity and shear stress of carotid artery duplex ultrasonography in patients with lacunar infarction
}

\author{
Seul-Ki Jeong ${ }^{1 *}$ and Robert S Rosenson ${ }^{2}$
}

\begin{abstract}
Background: This study describes a new method for determining site-specific vascular shear stress using dynamic measures of shear rate and blood viscosity (BV) in the carotid arteries, and examines characteristics of carotid arterial shear stress among patients with lacunar infarction.

Methods: Vascular shear stress measurements were conducted in 37 patients (17 lacunar infarction patients and 20 control subjects) using duplex ultrasonography. Vessel wall diameters and velocities were measured in each arterial segment at peak-systolic (PS) and end-diastolic (ED) phases, for calculation of PS/ED shear rates. PS/ED shear stresses $\left[d y n e / \mathrm{cm}^{2}\right]$ were determined with PS/ED shear rates and shear-rate dependent BV values. For comparison, both values of hematocrit-derived BV and BV measurements at $300 \mathrm{~s}^{-1}$ were used for calculation of shear stress.

Results: All cardiovascular disease (CVD) risk factors including BV values were similar between the two groups. In both common carotid arteries, PS and ED shear stresses were significantly lower in the patients with lacunar infarction than in controls in multivariate models that included age, sex, and other major CVD risk factors. PS and ED shear stresses using the shear rate specific BV were $4.5 \%$ lower and $7.3 \%$ higher than those using the two other $B V s$, respectively.

Conclusion: Lacunar infarction was associated with reduced carotid arterial shear stress. The use of estimated BV for calculating carotid arterial shear stress provides more accurate assessment of the hemodynamic contribution of shear stress than previous models that have arbitrarily assigned a constant value to this dynamic flow property.
\end{abstract}

Keywords: Vascular shear stress, Blood viscosity, Shear rate, Peak-systolic, End-diastolic

\section{Background}

The vascular system can generally be viewed as a closed system, constraining a series of biochemical processes and mechanical stresses that are regulated by various homeostatic mechanisms [1]. From this theoretical perspective, if mechanical stresses such as wall shear stresses and tensile stresses are not properly dispersed, then an artery may sustain injury. This mechanical injury ultimately results in atherothromboembolic disease and its clinical manifestations of stroke, myocardial infarction and claudication [2].

\footnotetext{
* Correspondence: jeongsk@jbnu.ac.kr

${ }^{1}$ Department of Neurology \& Research Institute of Clinical Medicine,

Chonbuk National University - Biomedical Research Institute of Chonbuk National University Hospital, San 2-20, Geumam-dong, Deokjin-gu, Jeonju, Jeonbuk 561-180, South Korea

Full list of author information is available at the end of the article
}

Blood is a fluid suspension of plasma and cells such as erythrocytes, leukocytes, and platelets, and it demonstrates non-Newtonian fluid mechanics. A non-Newtonian fluid has a variable, non-linear relation between blood viscosity and blood flow [3]. Specifically, blood viscosity is higher at low shear rates $(\dot{\gamma})$ and is reduced as shear rate increases [4]. This shear rate dependent aspect of blood viscosity $(\mathrm{BV}, \mu)$ has presented challenges to the accurate calculation of vascular wall shear stress, the frictional force per unit area acting tangentially to the arterial wall. To date, nearly all previous studies on arterial wall shear stress have neglected the flow-dependency of blood [5] and improperly presented BV as a constant Newtonian fluid [6-8]. Although the non-Newtonian characteristics of blood have been assumed in some previous studies, BV over ranges of shear

\section{() Biomed Central}

(c) 2013 Jeong and Rosenson; licensee BioMed Central Ltd. This is an Open Access article distributed under the terms of the Creative Commons Attribution License (http://creativecommons.org/licenses/by/2.0), which permits unrestricted use, distribution, and reproduction in any medium, provided the original work is properly cited. 
rates have not been measured directly, but the laws of viscosity were applied empirically $[9,10]$.

This study was designed to evaluate a new method for calculating vascular shear stresses along carotid arterial segments using duplex ultrasonography and dynamic BV measurements, and to examine whether the vascular shear stress of carotid artery was different between patients with lacunar infarction and control subjects using the new method (shear rate-specific BV and shear stress). This hypothesis is based on the observation that lacunar infarction is accompanied by endothelial dysfunction [11] and low cerebral arterial blood flow velocities [12]. Finally, we compared the shear stresses of the new method with those of the conventional methods using other BV determinations such as hematocritderived BV or constant values at shear rate of $300 \mathrm{~s}^{-1}$.

\section{Methods}

\section{Subjects}

Seventeen patients with lacunar infarction and 20 control subjects were examined for carotid duplex ultrasonography and measured for BV at the Department of Neurology, Chonbuk National University Hospital, Jeonju, South Korea. A diagnosis of lacunar infarction was made based upon acute onset of focal neurological deficit and a relevant lesion was seen on diffusion-weighted magnetic resonance imaging (MRI) of the brain [13]. A lacunar infarct was diagnosed when the brain MRI identified a lesion less than $15 \mathrm{~mm}$ in diameter with characteristics of high signal intensity on diffusion-weighted imaging and low signal intensity on the apparent diffusion coefficient map. The location of the lacunar infarction included either the basal ganglia, corona radiata, thalamus, or brainstem, but not cortex [12]. The control group was defined as patients with cardiovascular risk factors but no previous history of ischemic vascular diseases (e.g. stroke, myocardial ischemia, and lower extremity arterial disease). The institutional ethics committee at Chonbuk National University Hospital approved the present study. All subjects provided written informed consent.

\section{Assessments and measurements}

The medical history included data on prior CVD, type 2 diabetes mellitus, hypertension, dyslipidemia, and medication usage. Smoking status was recorded in pack-years. The patients were classified as hypertensive based on a persistent elevation of blood pressure $(\geq 140 / 90 \mathrm{mmHg})$ or treatment with antihypertensive medications. Type 2 diabetes mellitus was defined by fasting blood glucose $>7.0 \mathrm{mmol} / \mathrm{L}$ or previous use of glucose-lowering medications. Hypercholesterolemia was defined by a total cholesterol $>6.2 \mathrm{mmol} / \mathrm{L}$ or low-density lipoprotein cholesterol $>4.1 \mathrm{mmol} / \mathrm{L}$, or current use of cholesterollowering medication. Plasma concentration of total homocysteine (tHcy) was measured by fluorescence polarization immunoassay (AxSYM, Abbott Laboratories, Abbott Park, IL).

\section{Carotid ultrasonography}

In the present study, internal diameter, maximum centerline velocity, and intima-media thickness (IMT) were measured bilaterally along the common carotid artery (CCA), carotid bulb, and internal carotid artery (ICA), as shown in Figure 1. To calculate site-specific shear rates, diameters and velocities of both CCAs were used [14]. In the patients with lacunar infarction, carotid ultrasonography was performed at least one week after the vascular attack. A higher-frequency 5-to-12 MHz (12 L5) linear transducer (Terason t3000, Teratech, Inc, Burlington, MA) was used with ECG triggering, and all the examinations were performed by a certified neurosonologist (SKJ) who has performed more than 5,000 cases (10,000 carotid arteries) during the past 8 years. Intra-luminal diameters were measured separately at peak-systole (PS) and end-diastole (ED) using two-dimensionally guided continuous M-mode tracings of the intimal-luminal interface of the near and far walls of carotid arterial segments, as reported previously [14]. In all segments, video images of the interfaces between lumen and intima over 5 cardiac cycles were captured, stored, and the diameters were measured from fixed images. The axial resolution of the M-mode system was $0.1 \mathrm{~mm}$.

Velocities were measured at both PS and ED with the sample volume reduced to the smallest possible size $(1 \mathrm{~mm})$ and placed in the center of flow, thereby enabling the maximum centerline velocity to be determined. The Doppler angle was generally maintained between 45 and 55 degrees. The sample volume box was placed in the mid-lumen parallel to the vessel wall in all of the studies, as recommended [15].

The degree of ICA stenosis at both gray-scale and Doppler ultrasonography was stratified into the following 6 categories: (1) normal (no stenosis), (2) less than $50 \%$ stenosis, (3) $50 \sim 69 \%$ stenosis, (4) $\geq 70 \%$ stenosis to near occlusion, (5) near occlusion, and (6) total occlusion, as recommended [16].

\section{Exclusion criteria}

The patients with the following characteristics were excluded: 1) patients who showed ICA stenosis more than $50 \%$ in at least one side, 2) patients who have been performed for carotid artery revascularization including carotid endarterectomy or stenting. Finally, patients with lacunar infarction and control subjects, who had ICA of normal or less than $50 \%$ stenosis, were included in the present study. 

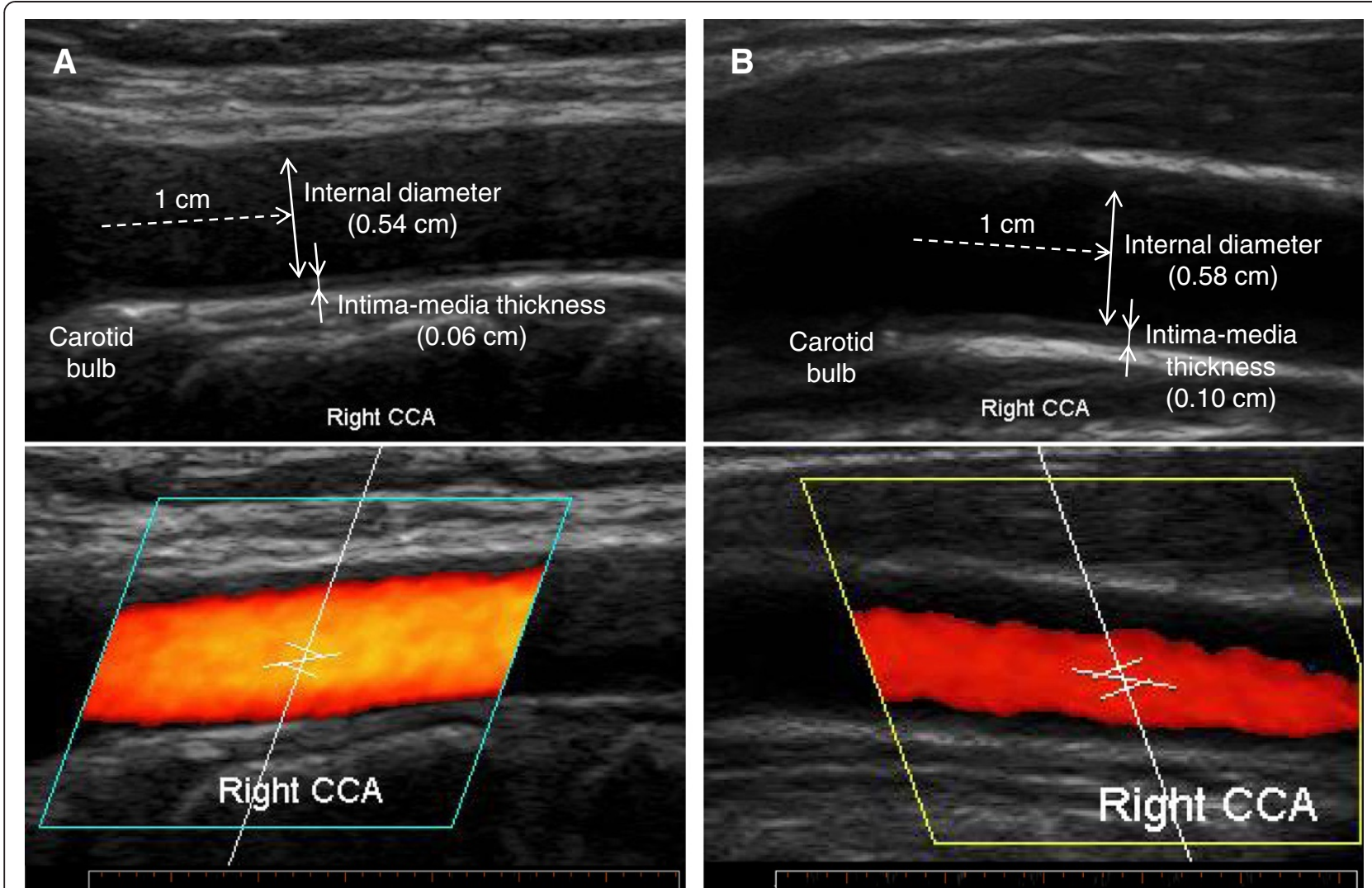

80. $\mathrm{PI} 1.28$ TAV $35.43 \mathrm{~cm}$ PSV $66.78 \mathrm{~cm} / \mathrm{s}$ EDV $21.30 \mathrm{~cm} / \mathrm{s}$
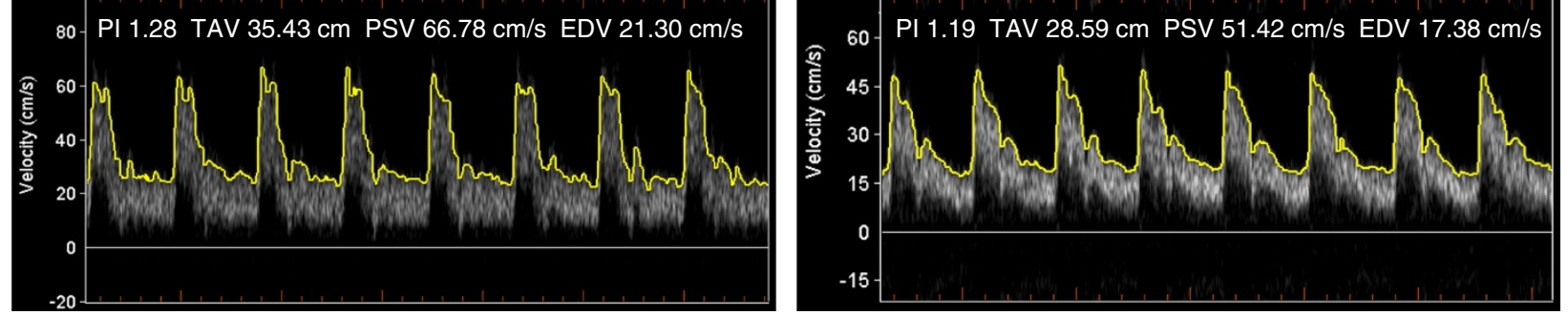

Figure 1 Representative ultrasonographic measurements of right common carotid internal diameters, intima-media thicknesses (IMTs), and blood flow velocities in a control subject (A) and a patient with lacunar infarction (B). Peak-systolic and end-diastolic internal diameters were measured at the segment $1 \mathrm{~cm}$ proximal to the beginning portion of carotid bulb, while IMT was measured at the peak of the R wave (end-diastole).

\section{Shear rate}

According to the PS/ED velocities and diameters along the carotid arteries, PS/ED shear rates were determined as follows [5]:

$$
\dot{\gamma}=4 \times \frac{V}{D}
$$

where $\mathrm{V}$ and $\mathrm{D}$ are the maximum centerline velocity and local lumen diameter, respectively. The PS and ED shear rates were calculated with corresponding PS/ED velocities and diameters, which were designated as PS shear rate $\left(\dot{\gamma}_{\mathrm{PS}}\right)$ and ED shear rate $\left(\dot{\gamma}_{\mathrm{ED}}\right)$.

\section{Blood viscosity}

For a measurement of BV, blood was sampled at antecubital vein and stored in ethylenediaminetetraacetic acid (EDTA) tube. BV was measured using a computerized scanning capillary viscometer (Hemathix ${ }^{\circ}$, Health Onvector, NJ) immediately after the carotid ultrasonography. The scanning capillary viscometer computes fluid viscosity using the Casson equation [17], which provides a relationship between the shear stress $(\tau)$ and shear rate $(\dot{\gamma})[18,19]$. The wall shear stress and shear rate in the Casson equation were calculated from flow velocity and pressure drop measurements in a U-shaped capillary tube. Qualitatively, the fluid dynamic theory used in the scanning capillary viscometer is similar to the Poiseuille flow relation, which describes BV $(\mu)$ as:

$$
\mu=\frac{\pi d^{4} \triangle P}{128 Q L}
$$


where $Q$ is the volume flow rate of blood, $d$ is the inside diameter of a capillary tube, $\Delta \mathrm{P}$ is the pressure drop along the capillary tube length, $\mu$ is $\mathrm{BV}$, and $L$ is the length of the tube.

\section{Shear rate $(\dot{\gamma})$ specific blood viscosity}

Shear rate specific BV values were determined at the corresponding PS/ED shear rates (i.e., $\mu_{\mathrm{PS}}$ and $\mu_{\mathrm{ED}}$ ) in each carotid arterial segment using the two Casson model constants $\left(k\right.$ and $\left.\mathrm{T}_{\mathrm{y}}\right)$ :

$$
\text { Blood viscosity }(\mu)=\frac{\tau}{\dot{\gamma}}=\frac{\tau_{y}}{\dot{\gamma}}+k+2 \sqrt{\frac{k \cdot \tau_{y}}{\dot{\gamma}}}
$$

\section{Blood viscosity at shear rate of $300 \mathrm{~s}^{-1}$ and of hematocrit-derived}

Hematocrit-derived BV $\left(\mu_{\mathrm{Hct}}\right)$ was calculated using the formula [20]:

$$
\begin{aligned}
& B V\left(\mu_{H c t}, \text { centiPoise, } c P\right) \\
& \quad=1.4175+5.878 H-12.98 H^{2}+31.964 H^{3}
\end{aligned}
$$

where $\mathrm{H}$ meant hematocrit (\%)/100.

Values of BV at $300 \mathrm{~s}^{-1}\left(\mu_{300 \mathrm{~s}-1}\right)$ were chosen from the measurements using the computerized scanning capillary viscometer.

\section{Shear stress}

The wall shear stresses specific to PS/ED BV values (e.g., $\mu_{300 \mathrm{~s}-1}, \mu_{\mathrm{Hct}}$, and $\mu_{\mathrm{PS}}$ and $\left.\mu_{\mathrm{ED}}\right)$ and PS/ED shear rates were calculated as follows:

PS shear stress $\left(\tau_{P S}\right)=\dot{\gamma}_{P S} \times\left(\mu_{P S}\right.$ or $\mu_{300 \text { s- } 1}$ or $\left.\mu_{\mathrm{Hct}}\right)$ ED shear stress $\left(\tau_{E D}\right)=\dot{\gamma}_{E D} \times\left(\mu_{E D}\right.$ or $\mu_{300}$ s-1 or $\left.\mu_{\mathrm{Hct}}\right)$

\section{Statistical analysis}

Descriptive data for the major characteristics were expressed as means \pm standard deviations (SD) or percentage as appropriate. An independent $\mathrm{t}$-test was used to determine the statistical differences in the continuous variables, whereas a chi-square test was used for categorical variables. For comparison of adjusted PS/ED shear stresses, analysis of covariance was performed separately in the carotid segments and expressed as adjusted means \pm standard errors. The mean values of PS/ED shear stresses using 3 methods of BV determination were compared with paired t-test. All statistical analyses were conducted using PASW Statistics 18 (SPSS, Chicago, IL).

\section{Results}

Seventeen patients with lacunar infarction and 20 control subjects were enrolled, as shown in Table 1. All the demographic characteristics and major CVD risk factors were not different between cases and controls. Triglyceride and HDL cholesterol showed differences of borderline significance. Plasma tHcy, hematocrit, and blood viscosities of both calculated and measured were not significantly different.

Carotid IMT showed significantly higher values in patients with lacunar infarction than the control subjects only at the right bulb region, as shown in Table 2 . The left CCA also showed higher IMT values in the patients, which reached significance. On the contrary, all the PS and ED shear rates at the both-sided CCAs were significantly lower in the patients with lacunar infarction than the controls. The PS and ED shear stresses at the both CCAs using shear rate specific BVs were significantly lower in the patients with lacunar infarction than the controls, even adjusted for age, sex, and cardiovascular risk factors, as shown in Table 3. Shear stresses of the both CCAs using blood viscosities at $300 \mathrm{~s}^{-1}$ or hematocrit-derived BV also showed significant differences between the two groups. There was no significant difference of the PS and ED shear stresses at the both CCAs according to the laterality of lacunar infarction (data not shown).

PS and ED shear stresses of CCAs and blood viscosities (shear rate specific BV, BV at $300 \mathrm{~s}^{-1}$, and Hctderived BV) were measured, as shown in Table 4. Compared with the shear stresses using shear rate specific BV, overall $4.5 \%$ higher and 7.3\% lower PS and ED shear stresses were calculated using the two other BVs (Hct-derived BV or BV at $300 \mathrm{~s}^{-1}$ ). Blood viscosities

\begin{tabular}{|c|c|c|c|}
\hline & $\begin{array}{l}\text { Control } \\
(n=20)\end{array}$ & $\begin{array}{l}\text { Lacunar infarction } \\
\qquad(\mathrm{n}=17)\end{array}$ & $p^{*}$ \\
\hline Age, y & $64.7 \pm 8.0$ & $68.3 \pm 7.7$ & 0.181 \\
\hline Women,\% & 40.0 & 35.3 & 0.769 \\
\hline Smoker, ex- and current,\% & 25.0 & 41.2 & 0.295 \\
\hline Hypertension,\% & 65.0 & 64.7 & 0.985 \\
\hline Type 2 diabetes mellitus, $\%$ & 35.0 & 23.5 & 0.447 \\
\hline Hypercholesterolemia,\% & 45.0 & 23.5 & 0.173 \\
\hline Total cholesterol, mmol/L & $4.8 \pm 1.1$ & $4.6 \pm 0.9$ & 0.620 \\
\hline HDL cholesterol, mmol/L & $1.2 \pm 0.3$ & $1.0 \pm 0.3$ & 0.095 \\
\hline Triglyceride, mmol/L & $1.3 \pm 0.6$ & $2.4 \pm 3.0$ & 0.063 \\
\hline Plasma total Hcy, $\mu \mathrm{mol} / \mathrm{L}$ & $11.2 \pm 4.0$ & $12.3 \pm 4.4$ & 0.481 \\
\hline Hematocrit,\% & $42.0 \pm 5.0$ & $41.7 \pm 6.5$ & 0.892 \\
\hline Hematocrit-derived BV, CP & $4.0 \pm 0.6$ & $4.0 \pm 0.8$ & 0.953 \\
\hline$B V, c P, 5 s^{-1}$ & $11.2 \pm 2.4$ & $11.4 \pm 4.2$ & 0.840 \\
\hline $100 \mathrm{~s}^{-1}$ & $4.7 \pm 0.8$ & $5.0 \pm 1.3$ & 0.451 \\
\hline $300 s^{-1}$ & $4.0 \pm 0.7$ & $4.3 \pm 1.2$ & 0.364 \\
\hline
\end{tabular}
showed $0.9 \%$ and $13.0 \%$ differences for PS and ED

Table 1 Characteristics of subjects

Means \pm standard deviations (SD) or percentages were expressed. $\mathrm{HDL}$; high-density lipoprotein, Hcy; homocysteine, BV; blood viscosity. 
Table 2 Carotid intima-media thickness along carotid arteries, and peak-systolic (PS) and end-diastolic (ED) shear rates in both common carotid arteries

\begin{tabular}{cccc}
\hline & $\begin{array}{c}\text { Control } \\
(\mathbf{n}=\mathbf{2 0})\end{array}$ & $\begin{array}{c}\text { Lacunar infarction } \\
(\mathbf{n}=\mathbf{1 7})\end{array}$ & $\boldsymbol{p}^{\text {* }}$ \\
\hline Intima-media thickness, cm & & & \\
Right ICA & $0.9 \pm 0.3$ & $0.8 \pm 0.2$ & 0.243 \\
Bulb & $1.0 \pm 0.2$ & $1.2 \pm 0.2$ & 0.039 \\
CCA & $1.0 \pm 0.3$ & $1.1 \pm 0.3$ & 0.650 \\
Left ICA & $0.9 \pm 0.2$ & $0.8 \pm 0.2$ & 0.250 \\
Bulb & $1.1 \pm 0.3$ & $1.2 \pm 0.2$ & 0.141 \\
CCA & $1.0 \pm 0.2$ & $1.2 \pm 0.3$ & 0.051 \\
Shear rate, s ${ }^{-1}$ & & & \\
Right CCA, PS & $405.7 \pm 211.5$ & $288.4 \pm 68.5$ & 0.028 \\
ED & $142.9 \pm 98.8$ & $97.0 \pm 32.7$ & 0.062 \\
Left CCA, PS & $503.8 \pm 192.4$ & $330.8 \pm 109.9$ & 0.002 \\
ED & $201.2 \pm 117.2$ & $120.2 \pm 55.9$ & 0.013 \\
\hline
\end{tabular}

Means \pm SDs were expressed.

ICA; internal carotid artery, CCA; common carotid artery.

phases, respectively. There was no significant difference between BV of hematocrit-derived and BV at $300 \mathrm{~s}^{-1}$, and also for their calculated shear stresses.

\section{Discussion}

The present study introduces a novel method for calculating site-specific vascular shear at the CCA using dynamic measures of shear rate and BV. For the calculation, Casson model constants $\left(k\right.$ and $\tau_{y}$ ) were derived from BV data obtained using a computerized scanning capillary viscometer. In this study, we demonstrate that shear stress is a dynamic parameter that varies widely along the carotid artery. With the novel method, vascular shear stress at the both CCAs was significantly lower in patients with lacunar infarction than the control subjects. Although CVD risk factors were not significantly different between the two groups, and even carotid IMT showed variable findings, vascular shear stresses at the CCAs showed discriminative features. Low shear stress along carotid artery was reported to be associated with ischemic stroke of large artery atherosclerosis, especially in the same side to the affected hemisphere [21]. To our knowledge, this study is the first to compare vascular shear stress in the patients with lacunar infarction with control subjects, on a shear rate specific basis using the individual subject's own BV profile.

The vascular shear stress of the CCAs, irrespective of the methods of shear stress calculation, showed similar patterns of difference between the patients with lacunar infarction and controls, as shown in Table 3. But, there were significant differences for the mean values of CCA shear stresses and blood viscosities according to the
Table 3 Adjusted $^{*}$ (mean \pm SE) peak-systolic and end-diastolic shear stresses $\left(d y n e / \mathrm{cm}^{2}\right)$ of both common carotid arteries

\begin{tabular}{cccc}
\hline & $\begin{array}{c}\text { Control } \\
(\mathbf{n}=\mathbf{2 0})\end{array}$ & $\begin{array}{c}\text { Lacunar infarction } \\
(\mathbf{n}=\mathbf{1 7})\end{array}$ & $\boldsymbol{p}$ \\
\hline Shear stress using shear-rate specific BV & & \\
Right CCA, PS & $16.0 \pm 1.0$ & $12.2 \pm 1.1$ & 0.028 \\
ED & $6.2 \pm 0.4$ & $4.7 \pm 0.5$ & 0.044 \\
Left CCA, PS & $19.5 \pm 1.3$ & $13.0 \pm 1.3$ & 0.005 \\
ED & $9.1 \pm 0.9$ & $5.0 \pm 1.0$ & 0.010 \\
Shear stress using BV at 300 s ${ }^{-1}$ & & & \\
Right CCA, PS & $16.5 \pm 1.2$ & $12.0 \pm 1.2$ & 0.026 \\
ED & $5.7 \pm 0.5$ & $3.9 \pm 0.5$ & 0.041 \\
Left CCA, PS & $21.0 \pm 1.5$ & $12.7 \pm 1.7$ & 0.003 \\
ED & $8.9 \pm 1.0$ & $4.1 \pm 1.0$ & 0.008 \\
Shear stress using Hct-derived BV & & \\
Right CCA, PS & $16.6 \pm 1.3$ & $11.0 \pm 1.4$ & 0.013 \\
ED & $5.8 \pm 0.6$ & $3.6 \pm 0.6$ & 0.033 \\
Left CCA, PS & $20.8 \pm 1.6$ & $11.9 \pm 1.7$ & 0.003 \\
ED & $9.1 \pm 1.2$ & $3.8 \pm 1.2$ & 0.012 \\
\hline
\end{tabular}

${ }^{*}$ Adjusted for age, sex, smoking, hypertension, type 2 diabetes, hypercholesterolemia, hematocrit, plasma tHcy, total cholesterol, triglyceride, and HDL cholesterol.

application methods of BV. Using the shear rate specific $\mathrm{BV}$, the lowest values of BV were observed at the PS phase and the highest values were detected at the ED phase. As such, if blood is assumed to behave as a Newtonian fluid and a certain representative value is used, vascular shear stresses will be calculated as higher than their true values at the PS phases, and lower in the ED phases. In the present study, when shear rate specific BVs were used, PS and ED shear stresses were 4.5\% higher and 7.3\% lower than shear stresses using constant values of BV, as shown in Table 4. This discrepancy would become more pronounced in arterial segments with elevated pulsatility indexes [22]. While the diagnostic methods described herein require further validation, this rationale underscores the non-Newtonian behavior of blood flow and makes a meritorious case for further research.

Our study results are consistent with the report that lacunar infarction is associated with endothelial dysfunction [11], which is controlled by vascular shear stress [2]. Previously, we reported patients with lacunar infarction had lower blood flow velocities in cerebral arteries than patients with other ischemic stroke subtypes [12]. The both low vascular shear stress in the CCAs and low blood flow velocities in cerebral arteries of patients with lacunar infarction may foster understanding of the pathophysiology of the disease. 
Table 4 Comparisons of mean values ( \pm SD, $n=37$ ) of PS and ED shear stresses and BVs according to applications of shear rate specific BV (A), BV at $300 \mathrm{~s}^{-1}$ (B), and hematocrit-derived BV (C)

\begin{tabular}{|c|c|c|c|c|c|c|}
\hline & \multirow{2}{*}{$\begin{array}{c}\text { Shear rate- } \\
\text { specific BV (A) }\end{array}$} & \multirow{2}{*}{$\begin{array}{l}\text { BV at shear } \\
\text { rate } 300 \mathrm{~s}^{-1} \text { (B) }\end{array}$} & \multirow{2}{*}{$\begin{array}{l}\text { Hematocrit- } \\
\text { derived BV (C) }\end{array}$} & \multicolumn{3}{|c|}{$p^{*}$} \\
\hline & & & & A vs. $B$ & A vs. C & B vs. C \\
\hline \multicolumn{7}{|c|}{ Shear stress, dyne $/ \mathrm{cm}^{2}$} \\
\hline \multicolumn{7}{|c|}{ Right CCA } \\
\hline PS & $14.0 \pm 6.4$ & $14.4 \pm 7.2$ & $14.4 \pm 8.2$ & 0.047 & 0.605 & 0.753 \\
\hline ED & $5.5 \pm 3.0$ & $4.9 \pm 3.1$ & $5.0 \pm 3.6$ & $<0.001$ & 0.010 & 0.935 \\
\hline \multicolumn{7}{|c|}{ Left CCA } \\
\hline PS & $16.2 \pm 5.9$ & $17.1 \pm 6.8$ & $17.1 \pm 8.0$ & 0.003 & 0.244 & 0.695 \\
\hline ED & $7.0 \pm 3.7$ & $6.6 \pm 4.1$ & $6.7 \pm 4.9$ & 0.001 & 0.131 & 0.888 \\
\hline \multicolumn{7}{|c|}{ Blood viscosity, CP } \\
\hline \multicolumn{7}{|c|}{ Right CCA } \\
\hline PS & $4.1 \pm 1.0$ & $4.2 \pm 0.9$ & $4.0 \pm 0.7$ & 0.268 & 0.364 & 0.153 \\
\hline ED & $4.8 \pm 1.2$ & $4.2 \pm 0.9$ & $4.0 \pm 0.7$ & $<0.001$ & $<0.001$ & 0.153 \\
\hline \multicolumn{7}{|c|}{ Left CCA } \\
\hline PS & $4.0 \pm 1.0$ & $4.2 \pm 0.9$ & $4.0 \pm 0.7$ & 0.003 & 0.980 & 0.153 \\
\hline ED & $4.6 \pm 1.2$ & $4.2 \pm 0.9$ & $4.0 \pm 0.7$ & $<0.001$ & $<0.001$ & 0.153 \\
\hline
\end{tabular}

Low carotid arterial shear stress in the patients with lacunar infarction who have no obvious carotid stenosis suggests increased resistance in small cerebral arteries [23] that may potentially increase arterial pressure in the more proximate carotid arteries. The transmission of increased pressure drop of cerebral arteries would cause an increase of carotid arterial pressure, a reduction of flow velocity, a compensatory increase in arterial diameter, and a decrease of vascular shear stress. When the increase of arterial pressure and the decrease of shear stress reinforce themselves through a feedback loop toward greater instability, a vicious cycle might cause lacunar infarction. Although thrombosis due to endothelial denudation under high shear could occur [24,25], thrombosis in the milieu of low blood flow velocities and low shear stress may also increase the propensity for ischemic events, especially at vulnerable regions [26]. Under low shear, increased transudation of blood or its components in small arteries of the deep cerebral tissue might also occur through the increased porosity of gap junctions, the morphological changes of endothelial cells [27], to cause lacunar (small-sized) hemorrhage or potentially lacunar infarction [28].

The present study has several limitations. First, we used a local centerline velocity to determine a local shear rate, which is expressed as $4 \mathrm{~V} / \mathrm{D}$. This equation is derived from a parabolic velocity profile for a fully developed laminar flow in a straight circular tube [29]; so, the assumptions in this model have limitations for estimation of shear stress in regions of complex flow even in the relatively straight CCAs. However, the use of the centerline velocity together with $4 \mathrm{~V} / \mathrm{D}$ expression allows the estimation of local changes in the velocity gradient if there is no significant hemodynamic compromise [30]. Further investigations are required to determine if centerline arterial shear stress is a parameter that has some critical threshold values for vascular diseases or pathologic conditions. Our study showed 76 carotid arteries in patients with lacunar infarction at CCA peak systolic shear stress of $12 \sim 13 \mathrm{dyne} / \mathrm{cm}^{2}$, which is slightly lower than the previous report of carotid arterial shear stress in the subjects with high risk vascular profiles [30] or with carotid plaque [31]. Second, there were differences of shear rate and shear stress between the right and left CCAs. That might be largely due to the differences of cerebral arterial resistance, and in part, the proximal arterial geometry: the right CCA comes from a celiac trunk and might have more tortuosity than the left CCA which comes from aorta directly. Third, the present cross-sectional study could not address the causal relationship between low carotid artery shear stress and lacunar infarction.

\section{Conclusion}

We describe a new method to calculate dynamic vascular shear stress in human carotid arteries using flow rates obtained from ultrasonography and shear ratespecific BV. With the innovative method, the carotid arterial shear stress was significantly lower in patients with lacunar infarction than the control subjects. The prognostic contribution of decreased vascular shear stress to incident lacunar infarction requires further study. 


\section{Competing interest}

The authors declare that they have no competing interest.

\section{Authors' contributions}

SKJ participated in data collection, conceived the study, drafted and reviewed the manuscript. RSR conceived the present study and reviewed the manuscript. All authors read and approved the final manuscript.

\section{Acknowledgement}

This article was supported by Fund of Biomedical Research Institute of Chonbuk National University Hospital and the measurement of blood viscosity was supported by Medical Device Clinical Trial Center (MDCTC), Chonbuk National University Hospital.

\section{Author details}

${ }^{1}$ Department of Neurology \& Research Institute of Clinical Medicine, Chonbuk National University - Biomedical Research Institute of Chonbuk National University Hospital, San 2-20, Geumam-dong, Deokjin-gu, Jeonju, Jeonbuk 561-180, South Korea. ${ }^{2}$ Mount Sinai Heart, Mount Sinai School of Medicine, New York, NY, USA.

\section{Received: 10 April 2012 Accepted: 9 March 2013}

Published: 18 April 2013

\section{References}

1. Cunningham KS, Gotlieb Al: The role of shear stress in the pathogenesis of atherosclerosis. Lab Invest 2005, 85:9-23.

2. Cecchi E, Giglioli C, Valente S, Lazzeri C, Gensini GF, Abbate R, Mannini L: Role of hemodynamic shear stress in cardiovascular disease. Atherosclerosis 2010, 214:249-256.

3. Soulis JV, Farmakis TM, Giannoglou GD, Chatzizisis YS, Giannakoulas GA, Parcharidis GE, Louridas GE: Molecular viscosity in the normal left coronary arterial tree. Is it related to atherosclerosis? Angiology 2006, 57:33-40.

4. Fahraeus $R$, Lindqvist $\mathrm{T}$ : The viscosity of blood in narrow capillary tubes. Am J Physiol 1931, 96:562-568.

5. Cho Yl, Kensey KR: Effects of the non-Newtonian viscosity of blood on flows in a diseased arterial vessel. Part 1: Steady flows. Biorheology 1991, 28:241-262.

6. Efstathopoulos EP, Patatoukas G, Pantos I, Benekos O, Katritsis D, Kelekis NL: Wall shear stress calculation in ascending aorta using phase contrast magnetic resonance imaging. Investigating effective ways to calculate it in clinical practice. Phys Med 2008, 24:175-181.

7. Box FMA, van der Grond J, de Craen AJM, Palm-Meinders IH, van der Geest RJ, Jukema JW, Reiber JHC, van Buchem MA, Blauw GJ, for the PSG: Pravastatin decreases wall shear stress and blood velocity in the internal carotid artery without affecting flow volume: results from the PROSPER MRI Study. Stroke 2007, 38:1374-1376.

8. Groen HC, Gijsen FJ, van der Lugt A, Ferguson MS, Hatsukami TS, van der Steen AF, Yuan C, Wentzel JJ: Plaque rupture in the carotid artery is localized at the high shear stress region: a case report. Stroke 2007, 38:2379-2381.

9. Soulis JV, Giannoglou GD, Chatzizisis YS, Seralidou KV, Parcharidis GE, Louridas GE: Non-Newtonian models for molecular viscosity and wall shear stress in a 3D reconstructed human left coronary artery. Med Eng Phys 2008, 30:9-19.

10. Gijsen FJ, Wentzel JJ, Thury A, Mastik F, Schaar JA, Schuurbiers JC, Slager CJ, van der Giessen WJ, de Feyter PJ, van der Steen AF, et al: Strain distribution over plaques in human coronary arteries relates to shear stress. Am J Physiol Heart Circ Physiol 2008, 295:H1608-H1614.

11. Kim JS, Lee HS, Park HY, Kim SS, Kang HG, Kim NH, Park JS, Kim Y: Endothelial function in lacunar infarction: a comparison of lacunar infarction, cerebral atherosclerosis and control group. Cerebrovasc Dis 2009, 28:166-170.

12. Kim JT, Lee SH, Hur N, Jeong SK: Blood flow velocities of cerebral arteries in lacunar infarction and other ischemic strokes. J Neurol Sci 2011, 308:57-61.

13. Norrving B: Lacunar infarcts: no black holes in the brain are benign. Pract Neurol 2008, 8:222-228.
14. Jiang $Y$, Kohara K, Hiwada K: Association between risk factors for atherosclerosis and mechanical forces in carotid artery. Stroke 2000, 31:2319-2324.

15. Tahmasebpour HR, Buckley AR, Cooperberg PL, Fix CH: Sonographic examination of the carotid arteries. Radiographics 2005, 25:1561-1575.

16. Grant EG, Benson CB, Moneta GL, Alexandrov AV, Baker JD, Bluth El, Carroll BA, Eliasziw M, Gocke J, Hertzberg BS, et al: Carotid artery stenosis: gray-scale and Doppler US diagnosis-Society of Radiologists in Ultrasound Consensus Conference. Radiology 2003, 229:340-346.

17. Kim S, Cho Yl, Hogenauer WN, Kensey KR: A method of isolating surface tension and yield stress effects in a U-shaped scanning capillary-tube viscometer using a Casson model. J Non-Newtonian Fluid Mech 2002, 103:205-219.

18. Rosenson RS, Wolff D, Green D, Boss AH, Kensey KR: Aspirin. Aspirin does not alter native blood viscosity. J Thromb Haemost 2004, 2:340-341.

19. Jeong SK, Cho Yl, Duey M, Rosenson RS: Cardiovascular risks of anemia correction with erythrocyte stimulating agents: should blood viscosity be monitored for risk assessment? Cardiovasc Drugs Ther 2010, 24:151-160.

20. Guyton AC, Hall JE: Textbook of Medical Physiology. 10th edition. Philadelphia: W. B. Saunders; 2000.

21. Carallo C, Lucca LF, Ciamei M, Tucci S, de Franceschi MS: Wall shear stress is lower in the carotid artery responsible for a unilateral ischemic stroke. Atherosclerosis 2006, 185:108-113.

22. Lim MH, Cho Yl, Jeong SK: Homocysteine and pulsatility index of cerebral arteries. Stroke 2009, 40:3216-3220.

23. Kobayashi S, Okada K, Yamashita K: Incidence of silent lacunar lesion in normal adults and its relation to cerebral blood flow and risk factors. Stroke 1991, 22:1379-1383.

24. Fry DL: Acute vascular endothelial changes associated with Increased blood velocity gradients. Circ Res 1968, 22:165-197.

25. Li ZY, Howarth SP, Tang T, Gillard JH: How critical is fibrous cap thickness to carotid plaque stability? A flow-plaque interaction model. Stroke 2006, 37:1195-1199.

26. Nesbitt WS, Westein E, Tovar-Lopez FJ, Tolouei E, Mitchell A, Fu J, Carberry J, Fouras A, Jackson SP: A shear gradient-dependent platelet aggregation mechanism drives thrombus formation. Nat Med 2009, 15:665-673.

27. Levesque MJ, Liepsch D, Moravec S, Nerem RM: Correlation of endothelial cell shape and wall shear stress in a stenosed dog aorta. Arterioscler Thromb Vasc Biol 1986, 6:220-229.

28. Labovitz DL, Boden-Albala B, Hauser WA, Sacco RL: Lacunar infarct or deep intracerebral hemorrhage. Neurology 2007, 68:606-608.

29. Sutera SP, Skalak R: The history of Poiseuille's law. Annu Rev Fluid Mech 1993, 25:1-19.

30. Irace C, Cortese C, Fiaschi E, Carallo C, Farinaro E, Gnasso A: Wall shear stress is associated with intima-media thickness and carotid atherosclerosis in subjects at low coronary heart disease risk. Stroke 2004, 35:464-468.

31. Gnasso A, Irace C, Carallo C, De Franceschi MS, Motti C, Mattioli PL, Pujia A: In vivo association between low wall shear stress and plaque in subjects with asymmetrical carotid atherosclerosis. Stroke 1997, 28:993-998.

\section{doi:10.1186/1471-2377-13-36}

Cite this article as: Jeong and Rosenson: Shear rate specific blood viscosity and shear stress of carotid artery duplex ultrasonography in patients with lacunar infarction. BMC Neurology 2013 13:36. 